\title{
Observatorio
}

\section{"Gestión" de información personal, no sólo "recuperación" de información personal}

\author{
Por Deborah Barreau
}

Resumen: Se comparan las características y los comportamientos de la gente al clasificar y archivar objetos materiales y objetos digitales. Existe poca oferta de herramientas $-y$ también poco interés-para clasificar los objetos digitales de forma que posteriormente permitan una fácil interacción con ellos, así como reflexionar al revisarlos. Gracias al abaratamiento de las memorias informáticas se tiende a guardar todo sin ordenar, y se confía-erróneamente-sólo en un buen sistema de recuperación. Hay que investigar mucho más en los sistemas de gestión de información personal (PIM).

Palabras clave: Sistemas de gestión de información personal, Comportamiento, Objetos digitales, Documentos electrónicos, Abaratamiento del almacenamiento informático

Title: Personal information "management" (PIM), not just personal information "retrieval"

Abstract: Comparison of the characteristics and behaviour of people who are sorting and archiving physical and digital objects. There is limited selection -and also little interest-in

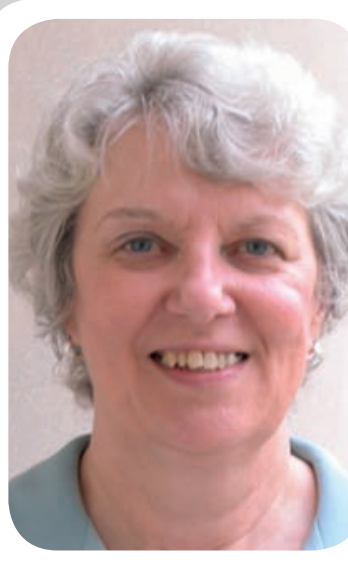

Deborah Barreau es profesora asociada en la School of Information and Library Science de la University of North Carolina at Chapel Hill donde imparte cursos de información en interacción humana y comportamiento organizacional. Su principal área de investigación se centra en las necesidades y usos de información en el lugar de trabajo, y en los efectos de la tecnología en la forma en que la gente trabaja, con especial énfasis en los comportamientos en la gestión de la información personal y las consecuencias de esos comportamientos para la gestión del conocimiento en las organizaciones. Es co-investigadora del proyecto Workforce Issues in Library Science (Wilis), financiado por IMLS. Se desempeñó como analista y bibliotecario de sistemas antes de obtener su doctorado en Estudios de Información por la University of Maryland en 1997. También tiene un master en Bibliotecología y grados BA y MAT en Sociología.

tools for classifying digital objects that subsequently allow easy interaction with them or mental reflection while reviewing them. Thanks to the availability of inexpensive computer memory, people tend to save everything without categorizing, and trust-erroneously-only in good retrieval skills. Much more research in personal information management systems (PIM) is needed.

Keywords: Personal information management systems, Behaviour, Digital objects, Electronic documents, Cheaper computer storage.

Barreau, Deborah. "Gestión" de información personal, no sólo "recuperación” de información personal”. El profesional de la información, 2009, julio-agosto, v. 18, n. 4, pp. 361-364.

DOI: 10.3145/epi.2009.jul.01

\section{LOS INVESTIGADORES HAN ESTUDIADO} los problemas de la gestión de la información personal (personal information management, PIM) durante más de veinticinco años.

Si bien ha mejorado la búsqueda en nuestro ordenador y navegar por los archivos personales es más fácil gracias a los thumbnails (imágenes minúsculas de un documento), los iconos, y las flexibles opciones de clasificación existentes, hemos avanzado poco en la resolución del problema básico de la gente de controlar su información personal.

Se ha puesto más atención en el desarrollo de herramientas de PIM que en la mejora de su práctica, en particular en lo que se refiere al mantenimiento, organi- zación, conservación, y uso de nuestros datos digitales. El almacenamiento barato y en cantidad ha alentado incluso los malos hábitos, pues no hay necesidad de filtrar, limpiar, manipular u organizar nuestros datos cuando tenemos tanto espacio disponible para almacenarlos y cuando tenemos buenas herramientas de búsqueda que pueden ayudarnos a encontrar lo que necesitamos rápidamente.

\section{"Con la memoria informática tan barata se prefiere almacenar todo en vez de decidir qué y cómo guardar"}


Por lo tanto, mantenemos las cosas porque hacerlo es más fácil que tomar una decisión sobre su valor potencial o su utilidad. Con el tiempo olvidamos lo que tenemos y ya no podemos distinguir fácilmente entre lo importante y lo inútil. Como consecuencia el resultado es a menudo hacer más copias de seguridad, pero no una conservación sistemática de lo que puede tener valor para un uso futuro.

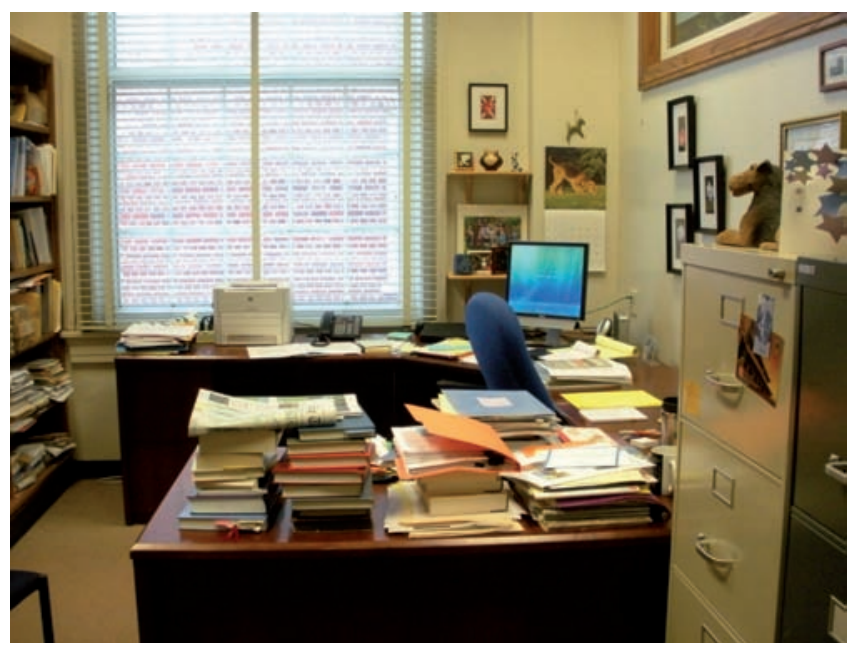

Despacho de la autora

\section{¿Es imposible imitar el entorno material?}

En el mundo físico interactuamos directamente con nuestras cosas. Son parte de nuestro entorno y la adquisición de nuevo material a menudo significa que debemos interactuar con lo que ya teníamos. Compramos marcos para las fotografías o reciclamos los viejos para poner en ellos las nuevas, almacenamos las fotos sustituidas en cajas, álbumes, o en otros lugares. Apilamos los nuevos libros en una mesa o corremos los de un estante para hacerles espacio, metemos documentos oficiales en carpetas, añadimos recetas a nuestras libretas de anillas, tomamos notas en los diarios y revistas, y colocamos los CDs y DVDs en armarios o en estantes según nuestros esquemas.

Cada interacción involucra de alguna manera procesos de pensamiento -tomamos decisiones sobre dónde colocar las cosas-, y en estos procesos tocamos las cosas que ya están ahí. El lugar elegido para dejar algo puede ser temporal, hasta que tengamos tiempo para acabar de decidir, pero las limitaciones del espacio físico y la "presión" que la vista de cosas acumuladas ejerce sobre nosotros nos obliga a tomar medidas al cabo de un tiempo. Cada contacto material es una oportunidad para la reflexión -nuestra memoria se estimula cuando vemos fotos antiguas, leemos nuestros informes médicos, revisamos recetas favoritas, o releemos anotaciones de nuestro diario- y los recuerdos nos hacen pensar. No se trata de encontrar algo que necesitamos, sino de redescubrir lo que tenemos.

Las opciones de que disponemos para trabajar con nuestros materiales digitales parecen más limitadas y menos satisfactorias. No estoy sugiriendo que no seamos reflexivos y cuidadosos cuando utilizamos el ordenador; sólo que los instrumentos que hemos creado o acumulado para el uso de la información digital limitan nuestras interacciones $\mathrm{y}$, por lo tanto, nuestra capacidad para descubrir y utilizar.

\section{"No existen programas que nos permitan ordenar y guardar los documentos digitales como hacemos con los materiales"}

Varios estudios han demostrado que las personas no son tan precisas cuando estiman la extensión de sus archivos digitales o a la hora de describir los contenidos de sus ficheros y directorios de ficheros. Aparte del nombre del fichero, tipo, tamaño, fecha de la última modificación, y los limitados elementos estructurales visibles en un thumbnail, sólo podemos descubrir el contenido de un objeto digital si lo abrimos. Cuando un directorio contiene cientos de archivos, la apertura de cada uno para saber su contenido no es práctica. Los diseñadores de sistemas de gestión de información personal saben que sólo buscamos para encontrar y recuperar nuestras cosas si tenemos una necesidad, pero también navegamos por nuestras cosas para reflexionar y recordar. Todavía no tenemos herramientas para realizar esto último de una forma cómoda.

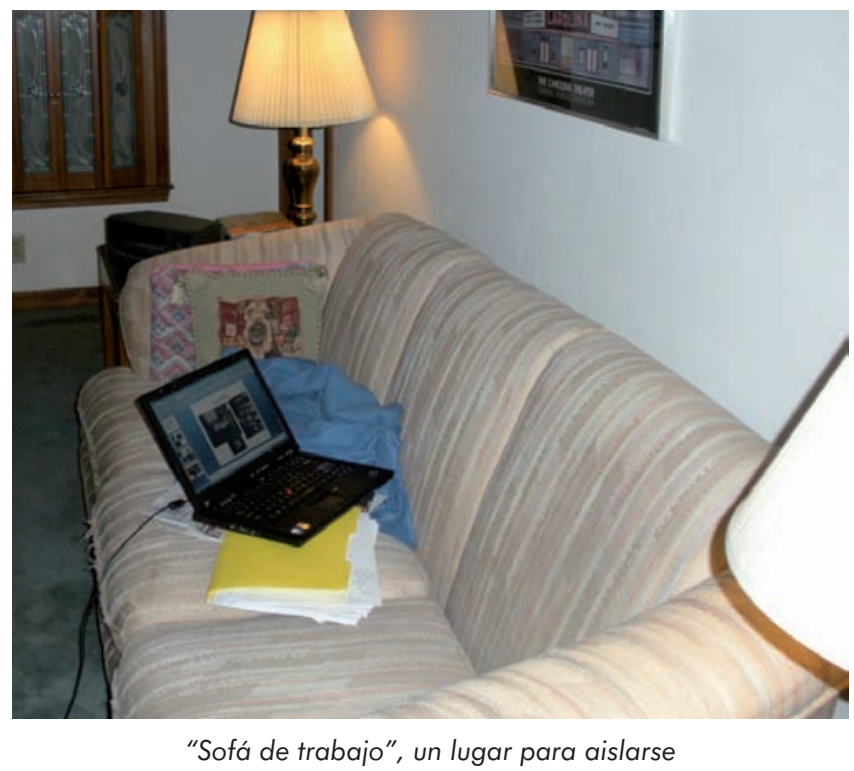




\section{¿Por qué nos cuesta tanto ordenar objetos digitales?}

Es poco probable que tengamos mejores herramientas para la gestión de nuestra información digital si no se investiga más para hallar la manera de ayudar a los usuarios a hacerse cargo de sus entornos digitales, de igual forma cómo lo hacen en el entorno físico. Ya desde el principio los investigadores descubrieron que los sistemas de gestión de información personal son relativamente superficiales y que las personas son renuentes a desarrollar y mantener esquemas rígidos para guardar sus ficheros.

Hemos interpretado que esto sugiere que la gente está demasiado ocupada, es demasiado vaga, demasiado desorganizada, o simplemente no quiere estructurar a conciencia su información, por lo que los desarrollos se ha centrado en la recuperación. Sin embargo, tal interpretación ignora la complejidad de nuestras relaciones con nuestras cosas personales, que es lo que hace que nos sea tan difícil categorizar.

Las fotografías digitales de la boda de un amigo, por ejemplo, pueden ser archivadas con la correspondencia del amigo o con otras fotografías de bodas a las que hemos asistido. Sin embargo, nuestros recuerdos de esa boda estarán vinculados a nuestras experiencias en la misma, tales como quiénes eran nuestros compa- ñeros, la comida que se sirvió, la música, el lugar de celebración del evento, e incluso el clima. Estas características pueden ser tan destacadas para nosotros como el propio acontecimiento. Así como podemos tener una gran variedad de 'tesoros' no vinculados entre sí en una caja de recuerdos, nuestros recuerdos de un evento pueden involucrar una variedad de asociaciones y reflexiones que se refieren tanto a otros eventos y experiencias en nuestras vidas como al que nos ocupa. Estas asociaciones hacen difícil asignar un objeto personal, ni siquiera la foto de la boda de un amigo, en una categoría específica.

Tenemos abundantes pruebas de que los usuarios están preocupados por sus prácticas de gestión de información personal. En 2004, Boardman y Sasse señalaron las dificultades para identificar o tratar de influir en las mejores prácticas para gestión de información personal, pero se dieron cuenta de que la investigación de conductas PIM tuvo el efecto de hacer a la gente más consciente de sus comportamientos y más decididos a mejorarlos. Y en toda la bibliografía PIM se hallan resultados similares. De hecho, la información personal que poseemos de nuestro entorno es tan única y nuestro comportamiento es tan individual que es difícil pensar que una propuesta práctica pudiera tener aplicación universal. Sin embargo, si analizamos los comportamientos PIM en contextos de trabajo o en

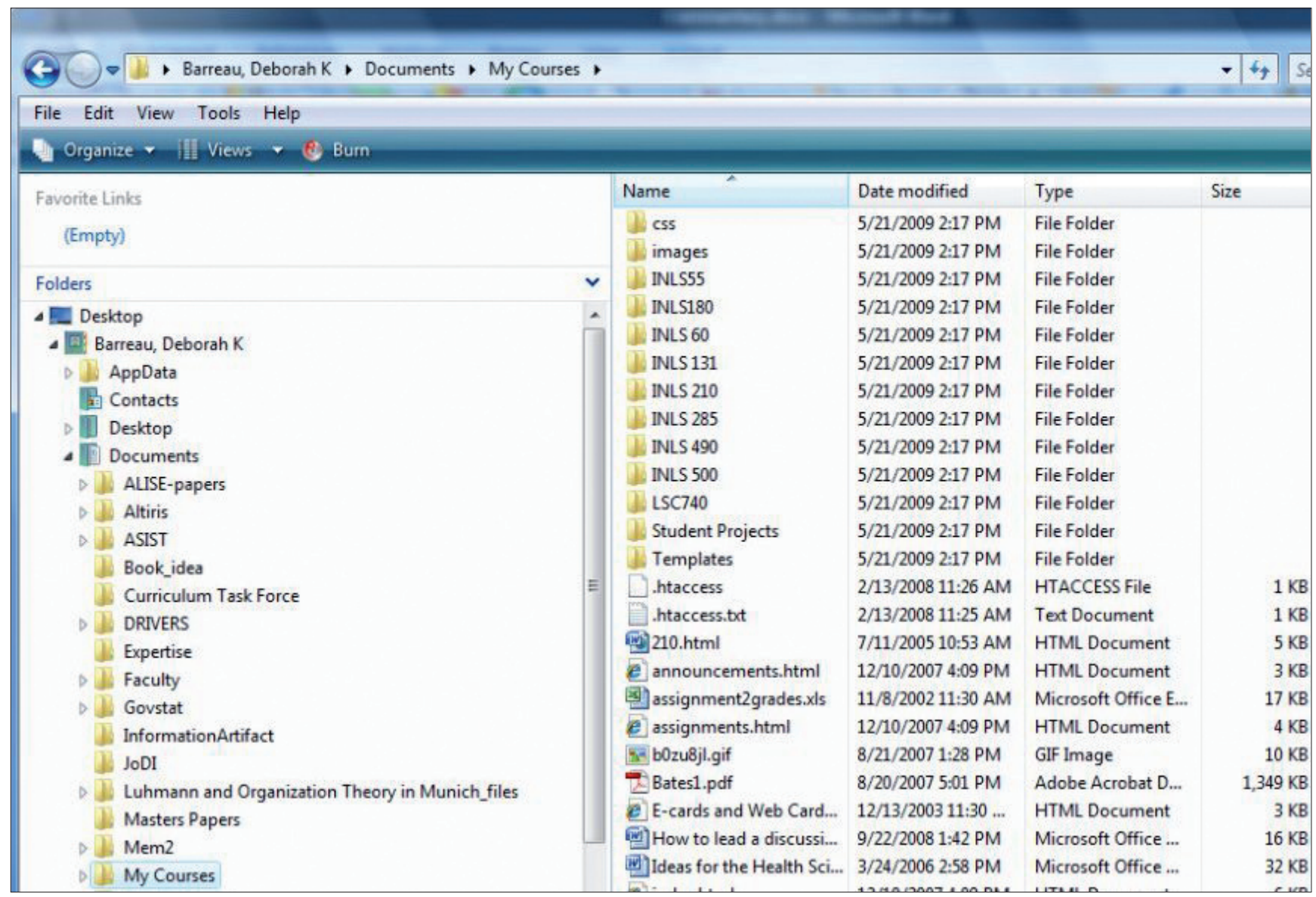

Típica organización de los archivos en un PC 
otros entornos compartidos, es posible que haya más oportunidades para generar cambios.

Algunos sugieren que en el futuro la generación de la Red estará mejor preparada para los retos de la gestión de la información digital personal, pero hay pocas pruebas que apoyen esta opinión. Otros sostienen que la verdadera ventaja de los entornos digitales sobre los materiales es que podemos guardar todo -no es necesario perder el tiempo en decidir qué y cómo organizar, ya que con unas mínimas capacidades de recuperación todo se hace accesible-.

Creo que esto es un error. Para mí, la necesidad de herramientas y de prácticas que apoyan la interacción y la reflexión sobre nuestro material es un reto importante que debe abordarse, en particular en los entornos educativos donde el pensamiento crítico es tan importante para el aprendizaje.

\section{"El diseño de PIMs requiere equipos de informáticos, psicólogos, pedagogos, sociólogos y documentalistas"}

Jerome Bruner y Lev Vygotsky, y más recientemente, Vera John-Steiner y Sherry Turkle, han explorado cómo nuestras cosas, tanto las que poseemos como las que creamos, estimulan y afectan la mente. Las herramientas para la interacción son útiles, pero seguramente los usuarios necesitarán además alguna orientación. Este es un problema que no puede ser resuelto sólo por los diseñadores de sistemas, sino que requiere el aporte de expertos tan variados como psicólogos, pedagogos, sociólogos de organización, y documentalistas. Lo más alentador es que la PIM es un área de investigación que sigue atrayendo a investigadores de diversas disciplinas y perspectivas. Hemos llegado lejos en la comprensión del comportamiento PIM, pero como comunidad pedagógica y de investigación tenemos mucho que hacer si queremos llegar a tener impacto y mejorar los hábitos.

\section{Bibliografía}

Barreau, Deborah K. "Context as a factor in personal information management systems". Journal of the American Society for Information Science, 1995 , v. 46, n. 5, pp. 327-339, June.

doi 10.1002/(SICI)1097-4571(199506)46:5<327::AID-ASI4>3.0.CO;2-C

Barreau, Deborah K.; Nardi, Bonnie A. "Finding and reminding: file organization from the desktop". ACM SIGCHI Bulletin, 1995, v. 27, n. 3, July, pp. 39-43.

Barreau, Deborah K. "The hidden costs of implementing and maintaining information systems". The bottom line: managing library finances, 2001, v. 14, n. 4, pp. 207-213.

Boardman, Richard; Sasse, Martina-Angela. "Stuff goes into the computer and doesn't come out": a cross-tool study of personal information management. In: Dykstra-Erickson, Elizabeth; Tscheligi, Manfred (eds.) Proceedings of ACM CHI 2004 Conference on human factors in computing systems, April 24-29, 2004, Vienna, Austria, pp. 583-590.

Bruner, Jerome Seymour. Actos de significado: más allá de la revolucion cognitiva. Madrid. 1995. 1a ed., 153 pp.

John-Steiner, Vera. Creative collaboration. New York: Oxford University Press, 2000, XIII, 259 pp. ISBN: 0-19-506794-0

John-Steiner, Vera. Notebooks of the mind: explorations of thinking, Rev. ed. New York: Oxford University Press, 1997, XXIII, 264 pp. ISBN: 0-19510896-5

Turkle, Sherry. La vida en la pantalla: la construcción de la identidad en la era de Internet. Barcelona: Paidós Ibérica, 1997, 414 pp. ISBN: 84-4930461-X

Turkle, Sherry (ed.) Evocative objects: things we think with. Cambridge, MA: MIT Press, c2007, ix, 385 pp.

eBook, Palo Alto, Calif.: ebrary, 2008, ISBN: 9780262201681

Vygotsky, Lev Semenovich (1896-1934). Pensamiento y lenguaje: cognición y desarrollo humano. Barcelona: Paidós, 1995, 237 pp.

Deborah Barreau, associate professor, School of Information and Library Science, University of North Carolina at Chapel Hill

barreau@email.unc.edu

\section{Suscripciones}

Renovar (o comenzar) la suscripción a "El profesional de la información" es ágil y sencillo.

Usted puede gestionar online su suscripción conectándose a esta web: http://www.elprofesionaldelainformacion.com/suscripciones. $h t m /$

Si lo desea puede comunicar con nosotros dirigiéndose a: suscripciones@elprofesionaldelainformacion.com

o al teléfono: +34-609352954 